\title{
RANSA: EXPORTACIÓN DE CONOCIMIENTOS LOGÍSTICOS
}

\section{Emilio Fantozzi Temple}

\author{
Gerente general de Ransa Comercial \\ Presidente de la Asociación Peruana de \\ Profesionales en Logística (Appprolog)
}

\section{Resumen}

La internacionalización es una etapa importante en la estrategia de crecimiento de las organizaciones líderes. Este fue un objetivo del plan de crecimiento de Ransa desde su fundación en 1939, que se puso en marcha en el 2003. Primero, de la mano de nuestros socios estratégicos, a los que ya atendíamos en el Perú, y luego a través de una adquisición en Centroamérica, que se concretó en el 2004 y que ha significado la oportunidad para obtener presencia en toda esa región.

Internacionalizar una empresa de servicios no es tarea sencilla. Aquí la estrategia y su implementación resultan claves. El presente artículo resume nuestro ingreso a los mercados de Bolivia, Ecuadory, en especial, al de El Salvador; así como la metodología que utilizamos para asegurarnos de que el proceso fuera exitoso, con énfasis en apalancar los conocimientos que ya habíamos desarrollado como organización y en la transmisión de los valores que forman parte de la cultura de Ransa. Creemos que estos han sido determinantes en los resultados que hemos alcanzado.

Palabras clave:

Internacionalización, estrategia empresarial, evaluación de nuevos mercados, choque cultural, exportación de conocimientos, servicios logísticos y valores. 


\section{Introducción}

Este artículo presenta el proceso que siguió nuestra organización para alcanzar sus metas y posicionarse con éxito en nuevos mercados, en especial en Centroamérica, donde se desarrolló una metodología bastante completa. El primer apartado trata acerca de la historia de Ransa en el Perú y sus primeros pasos en Bolivia y Ecuador. En el segundo se profundiza en la metodología utilizada para evaluar la oportunidad de crecimiento en Centroamérica; mientras que el tercero aborda la estrategia que se desarrolló en la práctica. Tras ello, exponemos cómo el componente cultural ha sido clave en este proceso.

\section{Antecedentes y primeros pasos internacionales}

Ransa fue fundada en 1939, por la empresa norteamericana Anderson Clayton \& Co., bajo el nombre de Reprensa Algodonera y Almacén Nacional S.A. Posteriormente, en 1970, pasamos a formar parte del Grupo Romero, y en 1974 tomamos el nombre por el cual se nos conoce en la actualidad.

Prestamos servicios logísticos que incluyen almacenaje, agenciamiento de aduanas, transporte, distribución horizontal y servicios a navieras. Asimismo, poseemos alianzas con operadores globales de comercio exterior para proveer una solución total de logística a nuestros clientes. Complementariamente, brindamos los servicios de procesamiento, packing, refrigeración y congelamiento de toda clase de productos alimenticios.

Nuestras operaciones en el ámbito nacional están diversificadas y especializadas, pues atendemos a clientes de los sectores de minería y energía, consumo masivo y retail, industria, agroindustria, pesquería, telecomunicaciones, automotriz, entre otros. Para ello contamos con un área de almacenamiento para carga seca en general de 340,000 m², así como capacidad para almacenar 280,000 toneladas de granos, camiones para transporte de carga pesada y furgones para distribución de mercadería en las ciudades, frigoríficos con capacidad para almacenar 8,000 toneladas, túneles de congelamiento, entre otras facilidades.

Y si bien nuestra presencia en el mercado peruano es de larga data, la internacionalización es una etapa relativamente reciente. De la mano de clientes que quisieron llevar nuestros conocimientos a otros mercados, ingresamos el 2003 a Bolivia, con una presencia importante en las 
ciudades de La Paz, Cochabamba y Santa Cruz. Un año después ingresamos a Ecuador, atendiendo a clientes del rubro de consumo masivo, brindando servicios de almacenaje y distribución horizontal, además de otros servicios especializados, como la administración de archivos.

\section{La experiencia de Centroamérica}

En octubre del 2003 se le presentó a Ransa la oportunidad de comprar en El Salvador la empresa almacenera Agdosa. Dicha adquisición se concretó en el 2004, por lo que se decidió hacer un estudio del mercado logístico de Centroamérica, que se constituyó en un ejercicio importante para identificar a los competidores y clientes potenciales en toda la región.

El estudio se realizó en tres etapas, entre los meses de marzo y julio del 2004. En la primera etapa se analizó la oferta del mercado, se alineó la visión del proyecto, tomando conocimiento de los operadores logísticos existentes, visitando los diferentes países de la región, evaluando la situación de la oferta de servicios logísticos en la zona y determinando un mapa preliminar de fortalezas, debilidades, riesgos y oportunidades.

La segunda etapa tuvo como foco la demanda de servicios logísticos. En esta fase se visitó a los clientes potenciales, se ajustó el mapa estratégico inicial, se detectaron las oportunidades para el corto plazo y se definieron segmentos de atención prioritaria en la estrategia.

Finalmente, en la última etapa del estudio se obtuvo como resultado la recomendación de segmentos meta y tipos de clientes prospecto, la propuesta inicial de servicios, la recomendación del tipo de socios y alianzas. Del mismo modo, se validó la factibilidad de la estrategia y se redactó un plan de negocios.

Así, el estudio reveló un ranking de los factores clave relacionados con el éxito de un operador logístico en Centroamérica, de mayor a menor importancia: experiencia en el sector, precios, conocimiento de los profesionales del operador logístico, infraestructura, alcances de los sistemas de información, integración de servicios, cobertura regional y servicio estandarizado.

Con ello se determinaron los servicios básicos que un operador logístico debía ofrecer, que incluían transporte terrestre internacional, aduanas, almacenaje, manejo de inventarios y preparación de pedidos, distribución horizontal, maquila y etiquetado. 


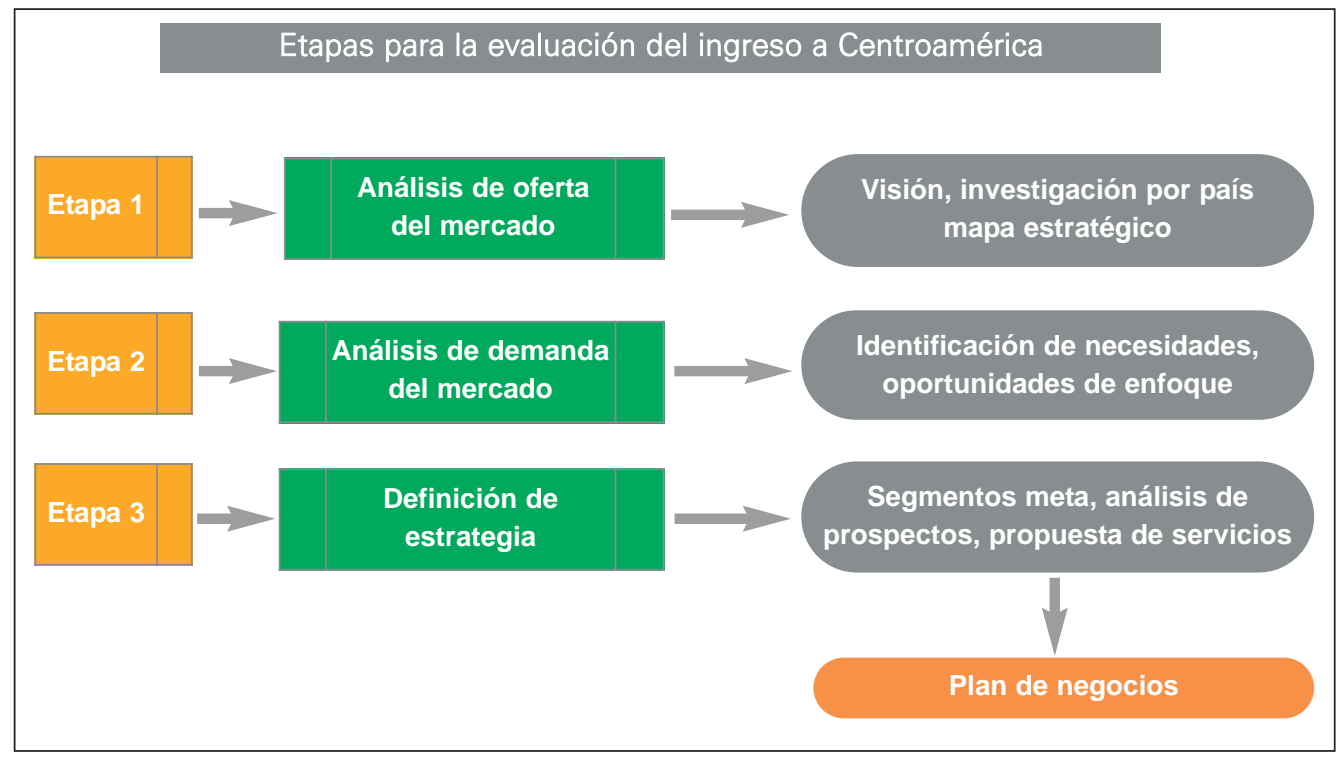

El análisis también reveló cuáles eran los sectores con mayor potencial a los cuales ofrecer servicios logísticos, entre los que se encontraban las compañías multinacionales manufactureras, los grandes minoristas regionales o locales, las empresas comercializadoras, los importadores y exportadores locales.

Asimismo, se identificaron los segmentos de mercado con mayor potencial y los de arranque inmediato, que conformaban las empresas de consumo masivo, supermercados, farmacéuticas y de electrodomésticos. Finalmente, el análisis incluyó la lista de clientes potenciales, señalando aquellos que se podían atender en el corto plazo.

De acuerdo con este análisis se determinaron dos tipos de contratos para la realización de servicios logísticos: de corto y de largo plazo. Los primeros se dan usualmente en operaciones logísticas de bajo valor agregado con commodities, donde el precio es muy importante y se revisa constantemente. Además, se trata de operaciones con una demanda muy volátil. Los segundos responden a soluciones logísticas personalizadas y dedicadas, asociadas a altas inversiones y donde el uso intensivo de la logística hace que el precio no sea lo más importante.

Tras ello se concluyó que el mercado de Centroamérica presentaba un importante potencial para un nuevo operador logístico, que aprovechara una oferta integrada de servicios y el crecimiento de la tercerización, así como el potencial que reportaba la firma de un Tratado de Libre Comercio con los Estados Unidos. 


\section{La estrategia en la práctica}

De este modo, en el 2005 se decidió constituir Ransa en El Salvador, de modo que Agdosa se posicionara en el segmento de precios y con el primer tipo de contratos, solo para el mercado salvadoreño; mientras que Ransa lo hacía en el segmento de alto valor agregado, que maneja contratos de largo plazo, y con miras a ser un operador logístico regional y líder en servicios y conocimiento.

Así, en Agdosa se mantuvo el statu quo, en el sentido de hacer solo pequeñas modificaciones en sus servicios, de modo que no se afectaran los precios. Sin embargo, Agdosa utilizaba ahora los sistemas de información y las mejores prácticas desarrolladas por Ransa. Además, ahora tenía la posibilidad de dar mayores servicios a sus clientes. Al mismo tiempo, Ransa se posicionaba como una marca premium en soluciones logísticas especializadas, con una fuerte promoción que incluía los siguientes ámbitos:

- Desarrollo de una cultura logística en la región. A través de una membresía activa en asociaciones locales y regionales de logística, así como en programas de alta capacitación; el patrocinio de actividades dedicadas a promocionar el sector; participación en conferencias relacionadas con la actividad; publicación de artículos especializados en revistas y periódicos; y charlas en universidades e institutos para la formación de futuros profesionales.

- Publicidad. En revistas y periódicos especializados, desarrollo de una página web que imparta cultura logística, folletos y material de soporte para la venta.

- Comunicación. A través de un boletín y canales internos para la comunicación de novedades entre sus empleados.

- Posicionamiento. Mediante la participación en los principales eventos y actividades de logística realizados por importantes instituciones líderes en los diversos sectores económicos.

- Creación de fuertes relaciones con sus clientes. A través de la realización de actividades en conjunto con sus clientes y creando grupos de trabajo multifuncionales que permitan la generación de proyectos de mejora para los diversos sectores en los cuales se desarrollan.

- Estudios de mejora para la cadena de abastecimiento de sus clientes, entregados a los más altos niveles de las empresas. 
Esta última fue la estrategia de entrada para consolidar operaciones en la región. El primer caso fue Superselectos, el supermercado líder de El Salvador, que cuenta con 71 tiendas y ventas por US\$450 millones anuales, en un país que tiene el $50 \%$ de sus ventas canalizadas a través de los autoservicios.

Ransa realizó una consultoría en la que se identificaron ahorros importantes si es que Superselectos tercerizaba su operación logística. Para ello nos comprometimos a invertir US\$10 millones en un moderno centro de distribución de $18,000 \mathrm{~m}^{2}$ para centralizar a sus proveedores de productos no perecibles, además de ofrecerles sistemas de información e infraestructura de última generación para manejar su logística. Todo ello apalancado en el conocimiento desarrollado en el Perú al trabajar con Supermercados Peruanos, empresa que perteneció al grupo holandés Ahold y que hoy está en manos del Grupo Interbank.

Así, tras la consultoría, suscribimos un contrato por seis años y construimos un almacén que inició sus operaciones en marzo del 2006. Hasta hoy los resultados de este trabajo han sido alentadores: Superselectos registró una disminución de $45 \%$ en los días de inventario en las bodegas de sus tiendas, los días de inventario disminuyeron en $47 \%$ en los centros de distribución y la centralización ha ido aumentando gradualmente hasta llegar a valores cercanos al 84\%, con un abastecimiento diario a las tiendas. Ello ha originado una reducción de los costos de operación de nuestro cliente, mejorando sustancialmente la calidad del servicio. Así, por ejemplo, uno de sus principales proveedores reportó un aumento de $15 \%$ en sus ventas.



Foto aérea del centro de distribución de Nejapa, Ransa, en El Salvador. 
Dados los importantes beneficios que obtuvo Superselectos, esta cadena nos recomendó para hacer el mismo trabajo con Unisuper, un supermercado de Guatemala, que cuenta con 37 tiendas y con el cual también suscribimos un contrato similar al de Superselectos para el manejo de su logística. Es decir, el primer trabajo que hicimos sirvió para avanzar en el proceso de internacionalización que nos habíamos propuesto.

\section{El choque culltural}

Más allá del tema estratégico, la internacionalización de Ransa ha representado para nuestra organización un desafío en términos de recursos humanos*. En ese sentido, el ingreso a Bolivia, Ecuador, El Salvador y Guatemala ha demandado mucho trabajo y tiempo por parte de nuestros cuadros directivos para poder inculcar al nuevo personal que se integraba a Ransa, una cultura corporativa en la que prime la satisfacción del cliente, el desarrollo del personal y el fomento al trabajo en equipo.

$Y$ es que aunque el proceso de crecimiento de Ransa se ha dado en una región que comparte un origen y una lengua comunes, hay elementos que distinguen a cada país de América Latina y que hacen que la adaptación y recreación de nuestra cultura no sean sencillas ni cortas, que deben tomarse en cuenta en la planificación de un proceso de internacionalización y gestionar como aspectos claves para tener éxito y crecer.

Es por ello que la internacionalización de Ransa ha implicado que los cargos estratégicos en nuestras sucursales sean ocupados inicialmente por peruanos que estén presentes desde el comienzo de las operaciones en cada país y que cuenten con un alto nivel de expertise en los servicios ofrecidos a nuestros clientes.

Son estos ejecutivos, plenamente inmersos en la cultura de Ransa y del Grupo Romero, los encargados de transmitir un estilo gerencial enfocado en resultados y donde el servicio al cliente, sólidamente arraigado, permite innovar y desarrollar cambios estructurales importantes en una empresa y de manera coordinada; así como implementar un estilo con un fuerte énfasis en el control y en la búsqueda permanente de colaboradores que cumplan con el perfil definido para asegurar un nivel de servicio diferenciado y comprometidos con los objetivos de la organización.

* Esta sección ha sido tomada de Fantozzi, Emilio. "Un choque necesario". Perú Económico. Lima, agosto del 2002, pp. 5-6. 
Así, en Ransa se han adoptado prácticas deliberadas en pos de conseguir estos objetivos. Por ejemplo, en el nivel de los cargos gerenciales, la red de trabajo que nuestros ejecutivos han conformado nos ha permitido tener una misma línea en el estilo de trabajo. Los nuevos gerentes que ingresan a Ransa o los trabajadores que son promovidos a los cargos gerenciales se adaptan rápidamente a esta dinámica, coordinando o desarrollando proyectos conjuntos con sus colegas.

Asimismo, los gerentes que manejan los lineamientos, procedimientos y conocimientos de la cultura de Ransa trabajan muy de cerca y van formando a los jefes de segunda línea, quienes se convierten en los ejecutores finales de todas las actividades dentro del marco de las directivas generales de nuestra organización, hasta llegar al nivel operativo y finalizando el proceso con la supervisión de los gerentes en todo sentido.

$Y$, en general, se programan reuniones periódicas a nivel de todas las áreas, en las que se discuten y analizan los resultados obtenidos a la fecha y todos los asistentes proponemos ideas de mejora.

\section{A manera de conclusión}

Ransa cuenta hoy con cerca de 540 colaboradores trabajando fuera del Perú: 80 en Bolivia, 170 en el Ecuador, 250 en El Salvador y 40 en Guatemala, donde estimamos llegar a 100 para finales de este año.

Las ventas de nuestra empresa en el extranjero llegaron a US\$8 millones el año 2006 y representaron el 12\% de las ventas de la empresa matriz en el Perú. Para este año se proyecta llegar a US\$12 millones, lo que representaría un crecimiento de 50\% frente al año previo y un $16 \%$ de las ventas en el Perú.

Tenemos muchos proyectos en marcha y esperamos estar en más países de la región en los siguientes 24 meses. Nuestra principal carta de presentación es la de que brindamos un servicio de calidad, ayudando a nuestros clientes a tener una mejor logística y, por ende, a ser más competitivos.

Estos resultados no serían posibles de no haberse apoyado en el conocimiento que Ransa ha generado a lo largo de los años al proveer soluciones logísticas eficientes a nuestros clientes y contar con gente comprometida con el trabajo y que tiene pasión por la logística. 


\section{Bibliografía}

Apoyo y Asociados Internacionales. Reporte de riesgo del 2006. [en línea] < http://www.aai.com.pe/files/instituciones_no_financieras/ ransa_comercial/ca/ransa_ca.pdf $>$.

Ransa Comercial (Perú). [en línea] <http://www.ransa.net/> . 